\title{
Idiopathic Pulmonary Arteriovenous Malformation - A Rare Entity
}

\author{
Sanyukta Hepat ${ }^{1}$, Ruchita Kabra², Abhijit Wadekar³, Sourya Acharya ${ }^{4}$, Samarth Shukla ${ }^{5}$, Sunil Kumar ${ }^{6}$ \\ 1,2,3,4,6 Department of Medicine, Jawaharlal Nehru Medical College, Datta Meghe Institute of Medical Sciences \\ (Deemed to be University), Wardha, Maharashtra, India. ${ }^{5}$ Department of Pathology, Jawaharlal Nehru Medical \\ College, Datta Meghe Institute of Medical Sciences (Deemed to be University), Wardha, Maharashtra, India.
}

\section{INTRODUCTION}

Pulmonary arteriovenous malformation (PAVM) is one of the rare pulmonary vascular anomalies. Pulmonary arteriovenous malformation results in right to left shunt due to the abnormal communications between the pulmonary arteries and the pulmonary veins bypassing the normal capillary bed. ${ }^{1}$ This condition being rare could be easily missed, hence, it is essential for clinicians to suspect it based on the classical clinical features. This helps in early diagnosis and deciding further appropriate treatment option. Here we report the case of a patient affected by a large idiopathic pulmonary arteriovenous malformation in the right lung.

Most patients with pulmonary arteriovenous malformation are asymptomatic. This is due to the chronic compensation and secondary erythrocytic response. Dyspnoea due to PAVMs are a result of right-to-left shunt. Initial diagnostic tools include chest radiography and contrast enhanced computed tomography but the gold standard is pulmonary angiography. ${ }^{2}$

Because AVM has substantial morbidity rates associated with it, all patients with PAVMs who can undergo embolization should be treated with transcatheter embolization. In rest of the patients, surgical excision should be considered.

The main objective of this study was to highlight the early suspicion and diagnosis of pulmonary arteriovenous malformation as this is easily missed and leads to undue delay of treatment.

\section{PRESENTATION OF CASE}

A 48 years old non-diabetic, non-hypertensive female patient was admitted with chief complaints of breathlessness on exertion since one year which gradually progressed from modified medical research council (MMRC) grade 2 to grade 3. She also had platypnoea since 6 months. She gave no history of chest pain, palpitations, orthopnea, and paroxysmal nocturnal dyspnoea. There was no history of fever, cough, abdominal pain, bleeding manifestations, swelling of legs, giddiness and loss of consciousness.

In view of the current COVID 19 pandemic, patient's reverse transcription polymerase chain reaction (RT PCR) test was done which was negative and she was admitted in medicine intensive care unit (ICU). Patient's detailed examination was done. On general examination, patient was conscious and oriented, afebrile, pulse rate $-78 / \mathrm{min}$, blood pressure $-110 / 70 \mathrm{mmHg}$. Initially, pulse oximetry showed $76 \%$ saturation on room air, correcting to $94 \%$ with supplemental oxygen and arterial blood gas analysis demonstrated hypoxemia with a Pao2 level of $53.6 \mathrm{mmHg}$. Central cyanosis and clubbing were present. No signs of congestive cardiac failure were present. On systemic examination: Cardiovascular system - normal heart sounds heard; Respiratory system - continuous machinery murmur in the right interscapular and suprascapular region. Other systemic examination was normal.
Corresponding Author: Dr. Ruchita Kabra,

Department of Medicine, Jawaharlal Nehru Medical College, Datta Meghe Institute of Medical Sciences (Deemed to be University),

Wardha, Maharashtra, India.

E-mail: ruchitapkabra@gmail.com

DOI: $10.14260 / \mathrm{jemds} / 2021 / 730$

How to Cite This Article:

Hepat S, Kabra R, Wadekar A, et al. Idiopathic pulmonary arteriovenous malformation; a rare entity. $J$ Evolution Med Dent Sci 2021;10(41):3604-3606, DOI: 10.14260/jemds/2021/730

Submission 19-06-2021,

Peer Review 26-08-2021,

Acceptance 31-08-2021,

Published 11-10-2021.

Copyright (c) 2021 Sanyukta Hepat et al. This is an open access article distributed under Creative Commons Attribution License [Attribution 4.0 International (CC BY 4.0)] 

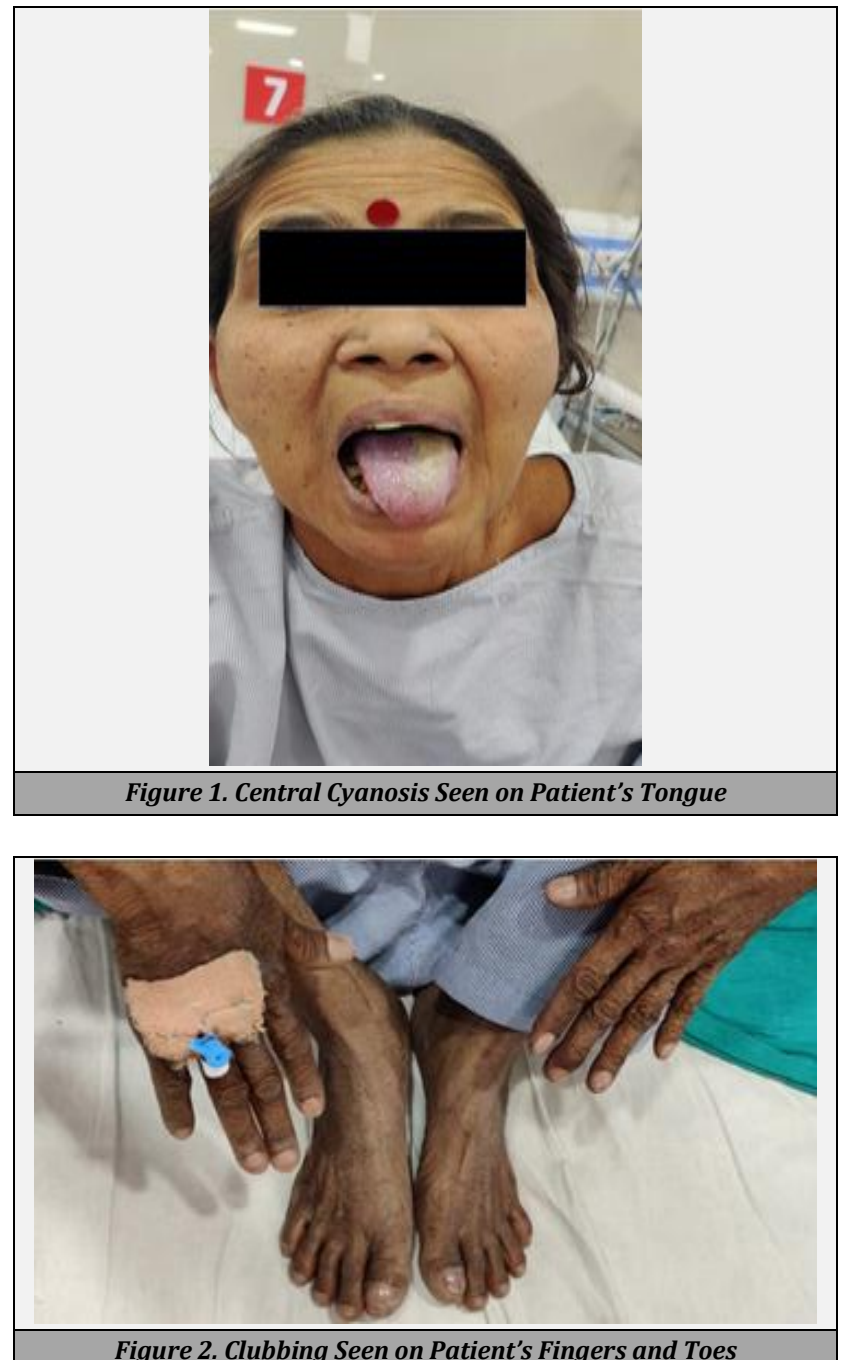

Laboratory investigations revealed her haemoglobin as $16.2 \mathrm{~g} / \mathrm{dL}$, total leukocyte count (TLC) 6,000/cumm and platelet count $1,20,000 /$ cumm. Peripheral smear showed predominantly normocytic, normochromic RBC's. Her prothrombin time international normalised ratio (PT INR) was 1.01, PT was 12.7 (PT control was 12.5) and activated partial thromboplastin time (APTT) was 30.4 (APTT control was 30). Her serum sodium was $136 \mathrm{mg} / \mathrm{dL}$, potassium was $4.0 \mathrm{mg} / \mathrm{dL}$. Liver enzymes were normal (Aspartate aminotransferase of $51 \mathrm{mg} / \mathrm{dL}$ and alanine aminotransferase of $31 \mathrm{mg} / \mathrm{dl}$, total bilirubin $0.9 \mathrm{mg} / \mathrm{dL}$, conjugated bilirubin $0.2 \mathrm{mg} / \mathrm{dl}$, unconjugated bilirubin $0.7 \mathrm{mg} / \mathrm{dL}$, albumin $3.5 \mathrm{~g} / \mathrm{dL}$, globulin $3.4 \mathrm{~g} / \mathrm{dL}$ ). Serum creatinine was $1.3 \mathrm{mg} / \mathrm{dL}$, urea was 40 $\mathrm{mg} / \mathrm{dL}$.

Electrocardiography showed normal sinus rhythm. 2D echo was done which showed normal study. In view of dyspnoea, cyanosis, clubbing, hypoxia, machinery murmur in the right interscapular and suprascapular region, pulmonary arteriovenous malformation was suspected, and CT pulmonary angiography was done.

CT angiogram of pulmonary arteries revealed a large A-V fistula in right upper lobe for which transcatheter coil embolization was done. (Figure 3)

Patient was thoroughly examined for telangiectatic lesions but could not find any lesion in nasal cavity, oral cavity or skin. She gave no history of epistaxis, spontaneous or traumatic. On enquiry, she denied history of visceral AVM's in other family members. As there was no evidence which suggest the diagnosis of hereditary haemorrhagic telangiectasia (HHT), this patient was supposed to have idiopathic pulmonary arteriovenous malformation.

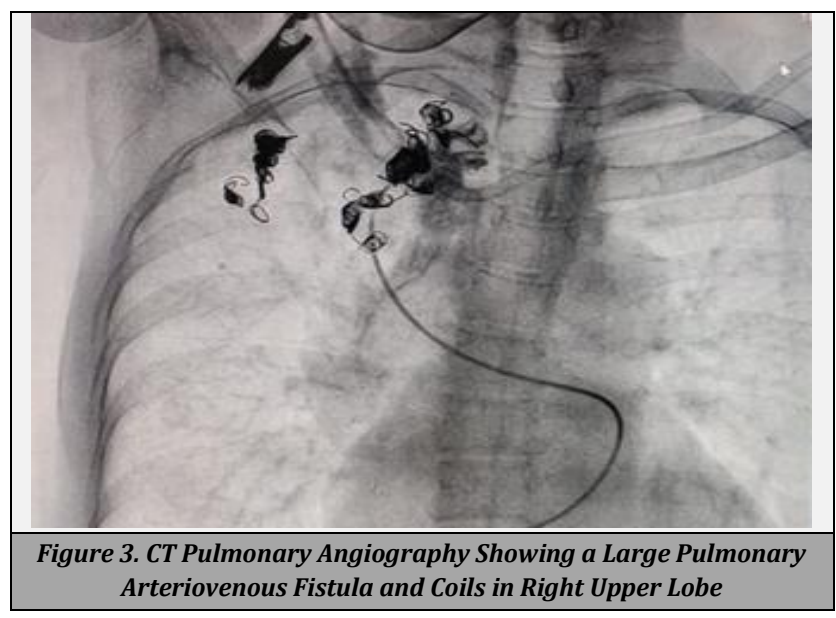

In the same setting, transcatheter coil embolization was planned. The patient underwent pulmonary angiography via right femoral vein, and a large pulmonary AVM was visualized in the right upper lobe. There were multiple small and large feeding arteries. A microcatheter was advanced and transcatheter embolization was performed on feeding arteries with multiple pushable micro coils of $12 \mathrm{~mm}$ X $14 \mathrm{~mm}$ two in number and $10 \mathrm{~mm} \mathrm{X} 14 \mathrm{~mm}$ two in number, total of 4 micro coils were placed and feeding arteries were successfully occluded. Post-embolization angiography was done and showed no more AV malformations and the procedure was completed.

There were no post-procedure complications seen, either it be vascular access site complication or contrast induced nephropathy. Post embolization patient's saturation improved to $90 \%$ on room air and arterial blood gas analysis demonstrated a Pao2 level of $84.5 \mathrm{mmHg}$.

HRCT thorax was done and was suggestive of metallic embolization coils noted in right upper lobe. (Figure 4)

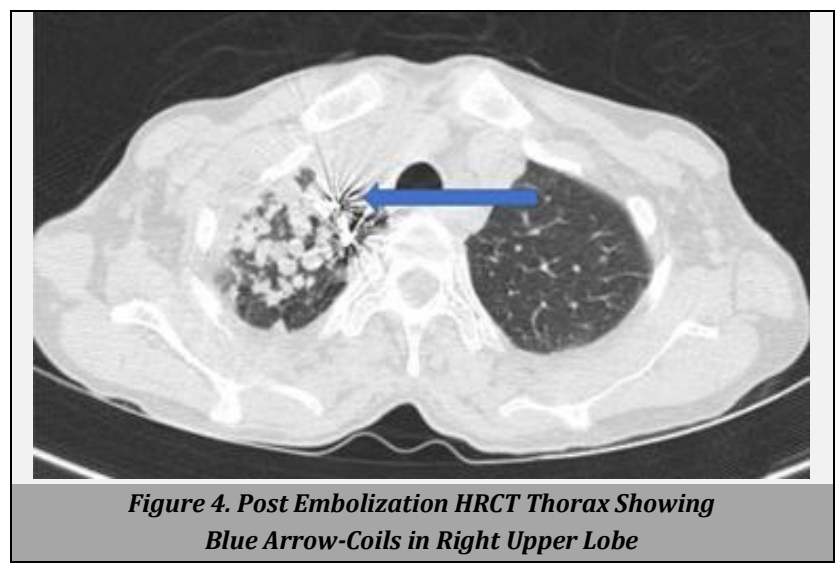

\section{DISCUSSION}

PAVM is an uncommon clinical problem. The classical triad of dyspnoea on exertion, cyanosis, and clubbing should alert the clinician to the possibility of a pulmonary AV malformation, as was seen in our case. PAVM are classified as simple or complex 
types. Single PAVM have a single feeding segmental artery leading to single draining pulmonary vein. About $80 \%$ of these malformations are of the single type and $20 \%$ are of the complex type. The complex type has two or more feeding arteries and veins. More than half of ACMs are seen in the lower lobes of the lung. The incidence of PAVM is $2-3$ per $1,00,00$ population. PAVMs have a slightly higher female preponderance. Hereditary haemorrhagic telangiectasia is common in most of the cases. ${ }^{3}$ When no obvious cause is found it is classified as idiopathic, as seen in our case.

The main pathophysiology is a right to left shunt. In most of the cases this does not cause a major hemodynamic upset; but patient may become symptomatic if the shunt is greater than $20 \%$ of the systemic cardiac output or if there is severe anaemia.

About $60 \%$ of the patients are asymptomatic but they may still have the signs of right to left shunt which includes exertional dyspnoea, cyanosis and clubbing and if severe, even haemoptysis. Up to $33 \%$ of the symptomatic patients go on to develop neurological complications. These may be due to rupture from cerebral PAVMs leading to intracranial haemorrhage. Half the lesions have audible vascular bruits, as was seen in our case. Hypoxia leading to polycythaemia can be masked if the patient has recurrent epistaxis due to telangiectasia. PAVMs can also cause brain abscess and this should be excluded. 4

Currently, the preferred management option is transcatheter embolization therapy or embolotherapy. Embolotherapy can be performed with coils or detachable balloons. This is usually carried out along with the investigative pulmonary angiography. After advancing the microcatheter, the feeding artery is occluded with coils. If multiple feeding arteries are present, they have to be occluded individually and may sometimes require more than one sitting. Surgery has now been superseded by embolization and is rarely required. 5 There is usually no periprocedural mortality with embolization of PAVM and complications are generally infrequent and self-limited. ${ }^{6}$

\section{CONCLUSIONS}

The most common presentation of PAVM is dyspnoea. The classical triad includes dyspnoea, cyanosis and clubbing. These findings in conjunction with continuous machinery murmur should alert the clinician towards the possibility of a PAVM causing right to left shunt. Treatment of PAVM with either surgery or embolotherapy is associated with minimal morbidity and virtually no mortality. ${ }^{6}$ Therefore, it is recommended that all symptomatic PAVM and PAVM more than $2 \mathrm{~cm}$ in diameter be treated with either surgery or embolotherapy. The preferred treatment option is embolotherapy as we can avoid the complications surrounding a major surgery.

Financial or other competing interests: None.

Disclosure forms provided by the authors are available with the full text of this article at jemds.com.

\section{REFERENCES}

[1] Park J, Kim HJ, Kim JM, et al. Successful treatment of a large pulmonary arteriovenous malformation by repeated coil embolization. Tuberc Respir Dis (Seoul) 2015;78(4):408-11.

[2] Khurshid L, Downie GH. Pulmonary arteriovenous malformation. Postgraduate Med J 2002;78(918):191-7.

[3] Kim HJ, Lee JS, Oh YM, et al. Clinical characteristics of pulmonary arteriovenous malformations in Koreans. Respirology 2015;20(1):155-9.

[4] Shovlin CL. Pulmonary arteriovenous malformations. Am J Respir Crit Care Med 2014;190 (11):1217-28.

[5] Shimohira M, Iwata K, Ohta K, et al. Hemoptysis due to pulmonary arteriovenous malformation after coil embolization during long-term follow-up. Case Rep Radiol 2019;2019:4506253.

[6] Gossage JR, Kanj G. Pulmonary arteriovenous malformations. A state of the art review. Am J Respir Crit Care Med 1998;158(2):643-61. 